Ionospheric effects are more noticeable on the intensity record as changes in the fading pattern. These recordings have been carried out consistently since the end of August, in which time the orbital plane has rotated more than $360^{\circ}$, so that each transit has been observed in both day and night conditions. Analyses are now in progress to examine the effects on the transmission of the variation in satellite height and of seasonal, diurnal and irregular changes in tho ionosphere.

This work is the result of a combined effort by the staff of this Laboratory.

G. H. Munro

L. H. Heisler

Sydney Laboratory,

Radio Research Board,

Commonwealth Scientific and

Industrial Research Organization,

Electrical Engineering Department,

University of Sydney. Jan. 23.

1 Murro, G. H., and White, R. B., Nature, 181, 104 (1958).

\section{The Samoan Artificial Aurora}

CULLINGTON $^{1}$ has recently reported the observation of an aurora-like phenomenon to the south-west of Apia (Samoa) which appears to have been produced by charged particles arriving along lines of force from the site of an atomic explosion, at great altitude in the neighbourhood of Johnston Island, some 2,000 miles distant. The interpretation of this phenomenon has been discussed in some detajl by Fowler and Waddington ${ }^{2}$ and by Kellogg, Ney and Winckler ${ }^{3}$. Since the particles are clearly guided by the Earth's magnetic field, the impact point for the particles, if we neglect the small drift in longitude, corresponds to the magnetic conjugate point for Johnston Island.

If the geomagnetic field was of purely dipole char. acter, this conjugate point could be obtained in a straightforward manner from geomagnetic co-ordinates. The two points would be on the same parallel of geomagnetic longitude and equidistant from, and on opposite sides of, the geomagnetic equator. However, it is well known from cosmic ray observations that the dipole representation is inadequate when considering the motion of charged particles in the Earth's field. Kellogg et al. have therefore calculated the expected position of the aurora on the assumption that the two conjugate points will be symmetrical about the so-called cosmic ray equator.

The behaviour of cosmic rays observed at the surface of the Earth is, however, determined by the geomagnetic field configuration all the way from the surface of the Earth to distances where the field ceases to be effective. The magnetic field lines connecting Johnston Island to Samoa, on the other hand, always lie within about 350 miles of the Earth's surface and consequently we should expect co-ordinate system based on the surface magnetic field parameters to be more appropriate than one dependent on cosmic-ray observations. We have therefore calculated the position of the conjugate point on the assumption that it should have a magnetic inclination of the same magnitude but opposite in sign to that of Johnston Island and that it should lie on the same magnetic meridian, defined by the direction of the horizontal component of the surface field. This

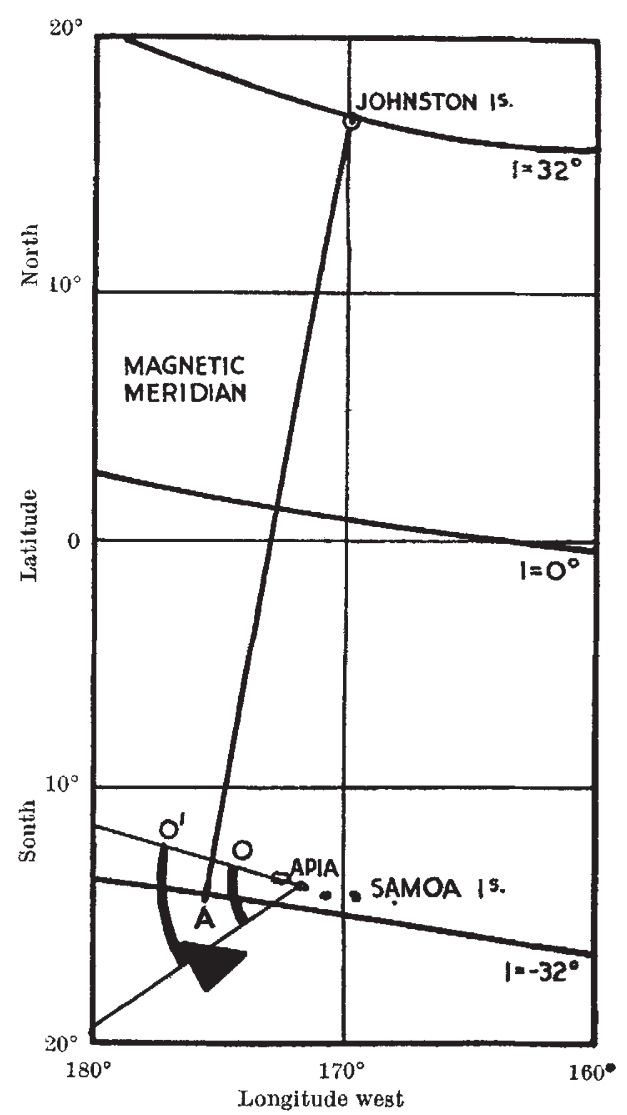

Fig. 1. $A$ is the position at which the aurora would be expected. and 100 miles. Kellogg et al.

point is marked $A$ in Fig. 1, which also shows the two lines of bearing between which the aurora was observed. The distance of the display from Apia is not known since the height of the display is uncertain. The elevation, however, is known to have been $12^{\circ}$ and the two thick lines $O$ and $O^{\prime}$ correspond to the position of the display assuming that the aurora occurred at a height of 50 miles and 100 miles respectively. The predicted point would correspond to a height of about 70 miles, which is the most frequent height of occurrence of genuine auroral displays at high latitudes.

The use of surface-field parameters clearly gives good agreement between the observed and calculated position of the aurora in this case. For high-latitude explosions, however, the guiding lines of force go out to great distances from the Earth and the surfacefield values are no longer appropriate. In calculating conjugate points for high-latitude events it is necessary instead to use the co-ordinate system adopted by Quenby and Webber ${ }^{4}$ to account for cosmic-ray phenomena.

Physics Department,

H. Elitiot

J. J. QUENBY

Imperial College of Science and Technology, London, S.W.7.

Feb. 17 .

'Cullington, A. L., Nature, 182, 1365 (1958).

Fowler, P., and Waddington, C. J., Nature, 182, 1728 (1958).

${ }^{8}$ Kellogg, P. J., Ney, E. P., and Winckler, J. R., Nature, 183, 358 (1259).

‘ Quenby, J. J., and Webber, W. R., Phil. Mag., 4, 90 (1959). 